Bio-grafia Escritos sobre la Biologia y su Enseñanza.

Edición Extra-Ordinaria. ISSN 2027-1034 P. p 805 - 813

Memorias del VII Encuentro Nacional de Experiencias en la Enseñanza de la

Biología y la Educación Ambiental y II Congreso Nacional de Investigación en la Enseñanza de la Biología

\title{
CONCEPCIONES ACERCA DE LOS TRABAJOS PRÁCTICOS DE FUTUROS DOCENTES DE CIENCIAS NATURALES Y EDUCACIÓN AMBIENTAL DE NEIVA
}

\section{CONCEPTIONS OF PRACTICAL WORK FUTURE TEACHERS OF SCIENCE AND ENVIRONMENTAL EDUCATION OF NEIVA}

\section{Elías Francisco Amórtegui Cedeño ${ }^{1}$}

\section{Resumen}

En este documento se presentan resultados preliminares acerca del estudio de las concepciones de futuros maestros del Programa de Licenciatura en Educación Básica con Énfasis en Ciencias Naturales Educación Ambiental de la Universidad Surcolombiana-USCO- (Neiva-Colombia). El estudio se llevó a cabo desde una perspectiva cualitativa, con base en el análisis de contenido y la recolección de información con base en la observación participante, el taller y el cuestionario. Los resultados muestran una tendencia marcada hacia ideas relacionadas con un enfoque didáctico tradicional con relación a la teoría-práctica, la imagen de práctica campo y la evaluación de este tipo de estrategias de enseñanza.

\begin{abstract}
This paper presents preliminary results on the study of future teachers' conceptions of the undergraduate program in Basic Education with Emphasis in Natural Sciences from the University Environmental Education Surcolombiana-USCO(Neiva-Colombia). The study was conducted from a qualitative perspective, based on content analysis and data collection based on participant observation, the workshop and the questionnaire. The results show a marked tendency toward ideas related to a traditional didactic approach in relation to the theory and practice, practice field image and evaluation of this type of teaching strategies.
\end{abstract}

Palabras Clave: Trabajo Práctico, Práctica de Campo, Práctica de Laboratorio, Concepciones.

Keywords: Practical work, Field Practice, Laboratory Practice, Conception.

\footnotetext{
${ }^{1}$ Docente de Planta Tiempo Completo. Programa de Licenciatura en Educación Básica con Énfasis en Ciencias Naturales y Educación Ambiental. Universidad Surcolombiana. Magister en Educación. elias.amortegui@usco.edu.co
} 
Bio-grafia Escritos sobre la Biología y su Enseñanza.

Edición Extra-Ordinaria. ISSN 2027-1034 P. p 805 - 813

Memorias del VII Encuentro Nacional de Experiencias en la Enseñanza de la

Biología y la Educación Ambiental y II Congreso Nacional de Investigación en la Enseñanza de la Biología

\section{Introducción}

Desde la perspectiva del Conocimiento Profesional del Profesor, las Prácticas de Campo y en general, los Trabajos Prácticos, son considerados estrategias de enseñanza, y desde esta perspectiva son un componente fundamental en el Conocimiento Didáctico del Contenido (Valbuena, 2007), sin embargo las investigaciones sobre el Conocimiento Profesional de Profesiones de Ciencias Naturales y Educación Ambiental son escasos y en su mayoría se han realizado respecto al conocimiento disciplinar que se enseña en cada una de las disciplinas, sean Física, Química o Biología (Subject matter Knowledge).

El Trabajo Práctico y la formación del profesorado en la enseñanza de las Ciencias han venido constituyéndose en una faceta muy valorada por los investigadores (Rodrigo et al, 1999). Específicamente las Prácticas de Campo (PC) se consideran importantes en la formación inicial de profesores de Ciencias (Dourado, 2006), al concebirse como una estrategia de enseñanza que permite relacionar la teoría con la práctica y favorecer el aprendizaje de diversos contenidos, constituyendo además una fuente de información directa, ejemplos y experiencias.

La revisión de antecedentes muestra que existe una gran cantidad de investigaciones sobre los Trabajos Prácticos en Ciencias, en mayor medida a partir de revisiones bibliográficas. Otro ámbito en el que se han centrado las investigaciones sobre los Trabajos Prácticos, ha sido particularmente las Prácticas de Laboratorio en secundaria y en educación superior con relación a concepciones de estudiantes, profesores y futuros profesores. Las investigaciones sobre las Prácticas de Campo son escasas.

En algunos casos, las investigaciones corresponden a las concepciones de profesores de ciencias naturales en educación primaria y secundaria, tanto a nivel anglosajón como latinoamericano. En una mayor proporción, las investigaciones sobre las Prácticas de Campo se han desarrollado en el área de la Geología, algunas corresponden a la implementación de estrategias de campo y enseñanza de esta disciplina y otras corresponden a la formación de profesores; inclusive se encuentran investigaciones relacionadas con la enseñanza de la Geografía. Por último, los estudios sobre Trabajos Prácticos y formación del profesorado de ciencias naturales son pocos, se destacan los trabajos con profesores de secundaria ciencias. Estos últimos han abordado las concepciones e ideas de los profesores en ejercicio durante sus clases de ciencias (Berezuki, Obara y Silva, 2009). 
Bio-grafia Escritos sobre la Biología y su Enseñanza.

Edición Extra-Ordinaria. ISSN 2027-1034 P. p 805 - 813

Memorias del VII Encuentro Nacional de Experiencias en la Enseñanza de la

Biología y la Educación Ambiental y II Congreso Nacional de Investigación en la Enseñanza de la Biología

Tan solo dos investigaciones han abordado la relación y el aporte de las Prácticas de Campo en la construcción del Conocimiento Profesional de profesores en formación inicial (Morcillo et al, 1999; Amórtegui y Correa, 2012) Lo anterior muestra el vacío que existe en este tipo de investigaciones, lo que genera la importancia de analizar el papel de las Prácticas de Campo en los programas curriculares de formación de docentes en Ciencias.

Esta revisión de antecedentes que han posibilitado construir un estado del arte, muestra que en términos generales los estudios sobre prácticas de campo se han centrado en cuatro tendencias. La primera corresponde a reflexiones o revisiones sobre dichas actividades, particularmente desde la perspectiva documental y en el contexto anglosajón.

La segunda tendencia corresponde a investigaciones cuyo objeto de estudio han sido la relación entre las prácticas de campo y la enseñanza de las ciencias naturales en general. Aquí tan solo se halla un trabajo que corresponde al diseño de una salida de campo (Alarcón y Piñeros, 1989).

La tercera tendencia corresponde a las concepciones sobre prácticas de campo en la enseñanza de las ciencias. Allí los trabajos han sido mayoritariamente desde una perspectiva cualitativa en donde ha predominado el uso de instrumentos como el cuestionario. Esta tendencia se ha visto marcada por su población objeto de estudio, pues en su mayoría ha correspondido a maestros en ejercicio en los niveles primarios y secundarios.

Por último, la cuarta tendencia corresponde a las investigaciones sobre prácticas de campo y su relación con el pensamiento-conocimiento del profesor. Tan solo dos investigaciones se han centrado en el contexto de la formación inicial de profesores de Biología y el aporte de las prácticas de campo a la construcción del Conocimiento Profesional del Profesor (Amórtegui, Gutiérrez y Medellín, 2009; Amórtegui y Correa, 2009). Sin embargo estas investigaciones no se han centrado en los procesos formativos y en las concepciones de los futuros maestros sobre dichas actividades.

\section{Metodología}

El estudio se llevó a cabo desde un enfoque cualitativo, empleando el método de análisis de contenido en el proceso de sistematización, y usando como herramientas de recolección de información el taller, la observación participante y un pequeño cuestionario que ha sido empleado en estudios anteriores por Amórtegui (2011). El grupo objeto de estudio consistió en 12 estudiantes entre 20 y 25 años de edad, pertenecientes en su mayoría a los estratos socioeconómicos 
Bio-grafía Escritos sobre la Biologia y su Enseñanza.

Edición Extra-Ordinaria. ISSN 2027-1034 P. p 805 - 813

Memorias del VII Encuentro Nacional de Experiencias en la Enseñanza de la

Biología y la Educación Ambiental y II Congreso Nacional de Investigación en la Enseñanza de La Biología

1,2 y 3 y que hacen parte del seminario de Didáctica I ofrecido en sexto semestre en el Programa de Licenciatura en Educación Básica con Énfasis en Ciencias Naturales y Educación Ambiental de la Universidad Surcolombiana-USCO (Neiva, Colombia). Las categorías de la investigación corresponden a lo planteado por Amórtegui y Correa (2012), y Puentes y Valbuena (2011), (Ver Tabla 1).

\begin{tabular}{|l|l|}
\hline Categoría & Subcategorías \\
\hline NATURALEZA & Imagen de práctica \\
\cline { 2 - 2 } & Relación teoría- práctica \\
\cline { 2 - 2 } & Clase de Trabajo Práctico \\
\hline FINALIDADES & \\
\hline METODOLOGÍA & Estructura de la Guía de Campo \\
\cline { 2 - 2 } & Enfoque didáctico \\
\cline { 2 - 2 } & Momento de realización \\
\cline { 2 - 2 } & Roles \\
\hline \multirow{2}{*}{ EVALUACIÓN } & Tipos \\
\cline { 2 - 2 } & Roles \\
\cline { 2 - 2 } $\begin{array}{l}\text { RELACIÓN Contenidos a evaluar } \\
\text { EPISTEMOLÓGICOS ASPECTOS }\end{array}$ & Biología como Ciencia \\
\cline { 2 - 2 } & Objeto de estudio de la Biología \\
\cline { 2 - 2 } & Producción de conocimiento biológico \\
\cline { 2 - 2 } & Imagen de Ciencia \\
\hline $\begin{array}{l}\text { ASPECTOS RELACIONADOS CON } \\
\text { LA FORMACIÓN DOCENTE }\end{array}$ & \\
\hline $\begin{array}{l}\text { ASPECTOS RELACIONADOS CON } \\
\text { EL CURRÍCULO DEL PCLB }\end{array}$ & \\
\hline
\end{tabular}

Tabla 1. Categorías de la investigación (Tomado de Amórtegui y Correa, 2012; Puentes y Valbuena, 2011).

\section{Resultados y discusión}

A continuación se presentan los hallazgos para cuatrosub categorías de investigación. En algunos casos se ilustra con declaraciones textuales de los futuros profesores de Ciencias Naturales y Educación Ambiental (se destaca la información relevante subrayando).

Para el caso de la Relación Teoría-Práctica, la mayoría de estudiantes consideran la Práctica como aplicación de la teoría y en ese sentido se caracteriza por ser de carácter unidireccional y se limita a contrastar, evidenciar y reforzar la teoría. 
Bio-grafia Escritos sobre la Biología y su Enseñanza.

Edición Extra-Ordinaria. ISSN 2027-1034 P. p 805 - 813

Memorias del VII Encuentro Nacional de Experiencias en la Enseñanza de la

Biología y la Educación Ambiental y II Congreso Nacional de Investigación en la Enseñanza de la Biología

E8.C1.1. "La práctica de campo es una actividad que se lleva a cabo fuera del aula de clase, donde se pone en práctica cada uno de los conocimientos adquiridos"

E3.C1.1. "La relación existente entre la práctica de laboratorio y la teoría es que aquella práctica es donde se van a aplicar los conocimientos adquiridos en la clase"

Esta concepción presenta elementos que se asocian teóricamente al modelo de Transmisión recepción planteado por Gil (1983), Perales (1994) y Baldaia (2006), quienes proponen dentro del modelo de transmisión recepción el Trabajo Práctico como complemento de la enseñanza teórica, que es transmitida por el docente, y que gracias a él podrá ser confirmada la temática trabajada. Según Baldaia (2006) bajo este modelo, el trabajo práctico asume un papel marcadamente demostrativo, en donde los conocimientos teóricos se confirman a través de actividades prácticas. Rodrigo et al (1999) plantea que bajo este modelo de transmisión, las actividades y experiencias de aula son escasas y se constituyen como la aplicación de la teoría. Esta concepción implica entonces una Trabajo Práctico cuya enseñanza está centrada en la exposición que realiza el profesor, en donde los alumnos redescubren conceptos y hechos y su participación se reduce a la toma de apuntes (Rodrigo et al, 1999).

Cabe resaltar que tan solo un estudiante concibe la relación teoría-práctica desde una perspectiva de Complementariedad.

E2.C1.1 "Debe ser una relación de complementariedad; la teoría debe hacerse práctica"

Esta idea podría asociarse al modelo constructivista planteado por Gil (1983), Perales (1994) y Baldaia (2006) en el cual se concibe el aprendizaje como un proceso dinámico y significativo, donde las actividades prácticas se realizan a través de la generación de conflictos, la resolución de problemas o por investigaciones.

En cuanto a las implicaciones en la formación docente, cabe resaltar que uno de los aspectos que genera mayor controversia está relacionado con las relaciones entre la teoría y la práctica, en general ambos elementos han sido considerados como dimensiones separadas pero siempre atribuyéndole superioridad a la teoría sobre la práctica. De este modo, es importante que en la formación docente se reflexione sobre la forma en que se aborda la teoría y la práctica, partiendo de la idea de tratarlas como campos mutuamente constituyentes y relacionados. Asimismo la relación entre teoría y práctica no supone que la teoría implique la 
Bio-grafia Escritos sobre la Biología y su Enseñanza.

Edición Extra-Ordinaria. ISSN 2027-1034 P. p 805 - 813

Memorias del VII Encuentro Nacional de Experiencias en la Enseñanza de la

Biología y la Educación Ambiental y II Congreso Nacional de Investigación en la Enseñanza de la Biología

práctica, se derive de ella o la refleje, sino que la teoría transforma la práctica y esta a su vez puede transformar la teoría (Moreno et al, 2006). En este mismo sentido, autores como Tamir (2005) plantean que la transición a la integración de la teoría con la práctica depende de las experiencias particulares, lo cual invita a los formadores de formadores a diseñar estrategias que favorezcan dicha transición.

Con relación a la categoría Imagen de Práctica, tres de los estudiantes se corresponden con una concepción de Práctica de Campo como Actividad en la cual los alumnos que asisten a ella deben poner a prueba los conocimientos teóricos adquiridos y tienen contacto con lo aprendido.

E10.C1.1: "Es una actividad dinámica en la que el estudiante tiene directamente el contacto con lo aprendido"

E7.C1.1: "Una práctica de campo es una actividad en donde los estudiantes ponen a prueba sus conocimientos teóricos"

Esta concepción puede implicar que las prácticas de campo no corresponden a actividades propias de la enseñanza, de la didáctica de las ciencias y particularmente del contexto escolar. Lo anterior podría estar relacionado con enfoques didácticos tradicionales en los que no se persiguen finalidades relacionadas con la construcción de conocimiento, en donde el papel del estudiante es totalmente pasivo, casi contemplativo (Rodrigo et al, 1999).

Este tipo de idea sobre una SC tiene más o menos una relación lejana con los contenidos escolares, por lo tanto se considera como una actividad desconectada y que no se encuentra integrada sobre una propuesta de enseñanza (Del Carmen y Pedrinaci, 1997).

Sería fundamental que los futuros maestros consideraran los Trabajos Prácticos como estrategias de enseñanza de las ciencias naturales; además implica reconocer un componente fundamental del Conocimiento Didáctico del Contenido, pues autores como Valbuena (2007), Carlsen (1999) y Magnusson, Krajcik y Borko (1999) y Park y Oliver (2008) identifican las estrategias de enseñanza como un aspecto fundamental en la estructuración del CDC.

Por último, con relación al Tipo de evaluación, la mayoría de estudiantes considera una evaluación de tipo Sumativa que consiste en la aplicación de conocimientos teóricos a la vida cotidiana. 
Bio-grafia Escritos sobre la Biología y su Enseñanza.

Edición Extra-Ordinaria. ISSN 2027-1034 P. p 805 - 813

Memorias del VII Encuentro Nacional de Experiencias en la Enseñanza de la

Biología y la Educación Ambiental y II Congreso Nacional de Investigación en la Enseñanza de la Biología

E5.C1.1. "La evaluación de una práctica de laboratorio consiste en la conceptualización de los instrumentos, sus usos, y la manera en la que los estudiantes pueden aplicar dichos conocimientos a la vida práctica.

Para Puentes (2008) dicha evaluación estaría relacionada con un enfoque tradicional en donde la evaluación consiste en una herramienta que permite al docente verificar y recibir de manos de los estudiantes la información que él ha transmitido o ha mostrado mediante un Trabajo Práctico, de manera que se privilegia una evaluación de contenidos sin tener en cuenta un proceso o el cambio de concepciones de los estudiantes. Para Jiménez (2000) la evaluación se centra en listas de conceptos, series, y experiencias prácticas que han partido de adoptar la SC como ilustración de la teoría en la que los alumnos siguen instrucciones detalladas. La evaluación equivale a examen, casi en exclusiva sobre el aprendizaje de hechos, conceptos y principios. Se mide el grado de aproximación entre sus formulaciones y las del profesor o el texto.

Haciendo referencia a los Contenidos a evaluar, priman en todos los estudiantes los de tipo Conceptual y Procedimental, en tanto se debe evaluar el manejo teórico de los temas y además las habilidades y procedimientos relacionados con el manejo de equipos e instrumentos de laboratorio.

\section{E4.C1.1: "La evaluación de una práctica de laboratorio consiste en la aplicación de los conocimientos teóricos y su comprensión, así como el debido manejo de los instrumentos y equipo de laboratorio"}

Lo anterior está relacionado por una parte con un enfoque didáctico tradicional en el que priman los conocimientos científicos y la comprobación de la teoría (Baldaia, 2006; Caamaño, 2003), pero además Trabajos Prácticos demostrativos en los que prima el aprendizaje de procedimientos, destrezas y habilidades como la observación, el registro, la toma de notas, de tal forma que estos se convierten en el centro de la evaluación (Caamaño, 2003; Rodrigo et al, 1999).

Desde la perspectiva de la construcción del Conocimiento Profesional del Profesor y el Conocimiento Didáctico del Contenido, a pesar de la poca explicitación de esta sub categoría, es fundamental que los futuros licenciados conciban no solo contenidos de enseñanza exclusivamente desde lo conceptual, sino que reconozcan la importancia de tener en cuenta la integración como contenidos de enseñanza tanto lo conceptual, lo procedimental y lo actitudinal (Valbuena, 2007). 
Bio-grafia Escritos sobre la Biología y su Enseñanza.

Edición Extra-Ordinaria. ISSN 2027-1034 P. p 805 - 813

Memorias del VII Encuentro Nacional de Experiencias en la Enseñanza de la Biología y la Educación Ambiental y II Congreso Nacional de Investigación en la Enseñanza de la Biología

\section{Conclusiones}

Con relación a las concepciones de los futuros maestros de Ciencias Naturales y Educación Ambiental cabe resaltar que la mayoría de ellos se encuentran en concepciones que se corresponden con enfoques didácticos tradicionales, en los que prima la corroboración de la teoría en la práctica, y la enseñanza aprendizaje de contenidos conceptuales y procedimentales.

Lo anterior muestra la importancia de favorecer procesos formativos en los cuales los estudiantes futuros maestros pongan en evidencia sus concepciones, pero además a través discusiones puedan detectarlas, modificarlas y re plantearlas en aras de una enseñanza-aprendizaje mucho más compleja y enriquecida desde perspectivas constructivistas.

\section{Bibliografía}

- Amórtegui, E; Gutiérrez, A; Medellín, F (2009). Las prácticas de campo en la construcción del conocimiento profesional de futuros profesores de Biología. En Bio-grafía, escritos sobre la biología y su enseñanza. 2 (1).En: http://www.pedagogica.edu.co/revistas/ojs/index.php/biografia/article/viewFile/ $\underline{160 / 129}$.

- Amórtegui, E Y Correa, M (2009). Las Prácticas de Campo Planificadas en el Proyecto Curricular de Licenciatura en Biología de la Universidad Pedagógica Nacional. Caracterización desde la perspectiva del Conocimiento Profesional del Profesor de Biología. Tesis para optar al título de Licenciado en Biología. Universidad Pedagógica Nacional.

- Amórtegui Elías y Valbuena Ussa Edgar (2010). Prácticas de campo en la enseñanza de la Biología y la formación docente: una revisión de antecedentes. IX Jornadas nacionales y IV Congreso internacional de enseñanza de la Biología. San Miguel de Tucumán 9 de octubre.

- Dourado, L (2006). Concepçoes e práticas dos profesores de Ciencias Naturais Relativas à implementaçao Integrada do Trabalho Laboratorial e do Trabalho de Campo. Revista Electrónica de Enseñanza de las Ciencias. 5 (1). Pp. 192-212.

- Porlán, R. Y Rivero, A. (1998). El conocimiento de los profesores: una propuesta formativa en el área de ciencias. Sevilla: Díada. 
Bio-grafia Escritos sobre la Biologia y su Enseñanza.

Edición Extra-Ordinaria. ISSN 2027-1034 P. p 805 - 813

Memorias del VII Encuentro Nacional de Experiencias en la Enseñanza de la

Biología y la Educación Ambiental y II Congreso Nacional de Investigación en la Enseñanza de La Biología

- Puentes, M. y Valbuena, E. (2011). Sistema de categorías para análisis didáctico de trabajos prácticos en la enseñanza de la Biología. Bio-grafía. Escritos sobre la Biología y su Enseñanza. (en prensa).

- Rodrigo, M; Morcillo, J; Borges, R; Calvo, M; Cordeiro, N; García, F; Raviolo, A (1999) Concepciones sobre el Trabajo Práctico de campo (TPc): una aproximación al pensamiento de los futuros profesores. Revista Complutense de Madrid. 10 (2), 261-285.

- Tardif, M. (2004). Los saberes del docente y su desarrollo profesional. Traducción de Pablo Manzano. Madrid: Narcea.

- Valbuena, E. (2007). El Conocimiento Didáctico del Contenido Biológico. Estudio de las concepciones disciplinares y didácticas de futuros docentes de la Universidad Pedagógica Nacional (Colombia). Tesis para optar al título de Doctor en Didáctica de las Ciencias Experimentales. Universidad Complutense de Madrid. 